\title{
Transcriptomics of liver and muscle in Holstein cows genetically divergent for fertility highlight differences in nutrient partitioning and inflammation processes
}

\author{
Bruce Moran ${ }^{1,2}$, Sean B. Cummins ${ }^{3}$, Christopher J. Creevey ${ }^{1,4}$ and Stephen T. Butler ${ }^{3^{*}}$
}

\begin{abstract}
Background: The transition between pregnancy and lactation is a major physiological change for dairy cows. Complex systemic and local processes involving regulation of energy balance, galactopoiesis, utilisation of body reserves, insulin resistance, resumption of oestrous cyclicity and involution of the uterus can affect animal productivity and hence farm profitability. Here we used an established Holstein dairy cow model of fertility that displayed genetic and phenotypic divergence in calving interval. Cows had similar genetic merit for milk production traits, but either very good genetic merit for fertility traits ('Fert+'; $n=8$ ) or very poor genetic merit for fertility traits ('Fert-'; $n=8$ ). We used RNA sequencing to investigate gene expression profiles in both liver and muscle tissue biopsies at three distinct time-points: late pregnancy, early lactation and mid lactation $(-18,1$ and 147 days relative to parturition, respectively).
\end{abstract}

Results: We found 807 and 815 unique genes to be differentially expressed in at least one time-point in liver and muscle respectively, of which $79 \%$ and $83 \%$ were only found in a single time-point; 40 and 41 genes were found differentially expressed at every time-point indicating possible systemic or chronic dysregulation. Functional annotation of all differentially expressed genes highlighted two physiological processes that were impacted at every time-point in the study, These were immune and inflammation, and metabolic, lipid and carbohydratebinding.

Conclusion: These pathways have previously been identified by other researchers. We show that several specific genes which are differentially regulated, including IGF-1, might impact dairy fertility. We postulate that an increased burden of reactive oxidation species, coupled with a chronic inflammatory state, might reduce dairy cow fertility in our model.

Keywords: Gene expression, Dairy cow fertility, Liver, muscle, Lactation

\section{Background}

The transition from late pregnancy to early lactation in dairy cattle has important implications for animal health, milk production and reproductive performance, and consequently the profitability of dairy farms $[1,2]$. The physiology of the transition dairy cow has been well studied; a variety of biological themes such as energy

\footnotetext{
* Correspondence: stephen.butler@teagasc.ie

${ }^{3}$ Teagasc, Animal \& Grassland Research and Innovation Centre, Moorepark, Fermoy, Co. Cork, Ireland

Full list of author information is available at the end of the article
}

balance, body reserve mobilisation, metabolic diseases, insulin resistance and immune function have all been found to be of importance [3-8]. Transcriptomics has been employed to aid identification of genes and networks that impact these various systems [9-11].

Hepatic gluconeogenesis, which supplies the vast majority of glucose utilized by ruminants, is of central importance in dairy cattle. The liver uses propionate (60-74 \%), L-lactate (16-26\%) and other minority precursors for glucose synthesis [5, 12, 13]. During late pregnancy, the glucose demands of the fetus account for 
up to $50 \%$ of total maternal glucose production [12]. During early lactation, high-producing dairy cows have a greater demand for glucose than can be met by gluconeogenesis from dietary sources alone. For example, dry matter intake (DMI) increases during the transition from late pregnancy to early lactation by 30 to $50 \%$, whereas mammary glucose requirements during early lactation are three times that of the uterus during late pregnancy [14]. Consequently, dairy cows enter negative energy balance (NEB), resulting in body reserve mobilisation. Insulin-sensitive tissues such as adipose and muscle become insulin resistant coincident with reduced circulating insulin concentrations. The combined effect of these changes is reduced peripheral tissue uptake of glucose, and hence greater glucose availability for the mammary gland. Local energy requirements in some tissues, for example muscle, can be met by the use of non-esterified fatty acids (NEFA) from adipose tissue lipolysis; the associated release of glycerol can be used by liver for gluconeogenesis [15].

Homeorhesis requires orchestrated changes across multiple tissues to support prioritization of nutrients for lactation $[12,16]$. The task of elucidating the physiological mechanisms underlying transition cow homeorhetic adaptations is ideally suited to transcriptomic analysis. Multiple tissues can be biopsied at multiple time-points to allow investigation of the genes involved in functional pathways and inter- and intra-cellular signalling. A lactating dairy cow genetic model of fertility has been developed and validated [17-19]. Cows have similar genetic merit for milk production traits, but either good (Fert+) or poor (Fert-) genetic merit for fertility traits. The physiological differences between the cows in this animal model have been extensively characterized [17-22]. We have taken the approach of sequencing the transcriptome of liver and muscle biopsies collected from Fert + and Fert- cows at three time-points corresponding to late pregnancy, early lactation and midlactation. Using bioinformatic methods to interrogate these data, we test the hypothesis that gene expression differences exist between cows with good or poor genetic merit for fertility traits during late pregnancy, early lactation, and mid-lactation in these tissues. In particular, the latter time-point coincides with the stage of lactation when pregnancy should be re-established and so is of economic importance. Although liver and muscle are not reproductive tissues, they are both important tissues for determining bioenergetic status, glucose production and utilization, and body reserve mobilization and accretion. Collectively, these physiological processes have implications for phenotypic fertility performance. Hence, transcriptomic profiling of liver and muscle tissue in cows genetically divergent for fertility traits could aid identification of physiological processes that underpin differences in phenotypic fertility performance.

\section{Methods}

\section{Lactating dairy cow model of fertility}

The cow model used in this study has been described previously [17]. Briefly, the national dairy cattle database of Ireland was screened for heifers due to calve for the first time in spring 2008. Restrictions were placed on the estimated breeding value (EBV) for milk production (between +200 and $+900 \mathrm{~kg}$ ) and proportion of Holstein genetics $(>75 \%)$. Within this population, heifers with extreme positive (i.e., poor fertility) and negative (i.e., good fertility) EBV for calving interval were identified. The Fert + animals represented the top $20 \%$ of the national herd in genetic merit for calving interval. Conversely, the Fert- animals represented the bottom $5 \%$ of the national herd in genetic merit for calving interval. In 2009, 16 animals $(n=8$ Fert + and $n=8$ Fert-) were enrolled in the current study. Animals were selected to maximize genetic diversity within both strains (i.e., different sires and maternal grand-sires) and to maximize differences between strains in the EBV for calving interval. In both Fert + and Fert- groups, the cows were a mixture of first $(n=2)$ and second $(n=6)$ parity Holstein animals (mean proportion of Holstein genetics $( \pm \mathrm{SD})=0.93( \pm 0.05))$, and were managed as a single herd.

\section{Animal characteristics}

The experimental procedures involving animals on this study were approved by the Teagasc Animal Ethics Committee and licensed by the Department of Health, Ireland, in accordance with the Cruelty to Animals Act (Ireland 1876) and the European Community Directive $86 / 609 /$ EEC. The animals were owned by Teagasc Moorepark, and all animals in the herd are routinely used for research purposes. Milk production was recorded daily, body weight was recorded weekly, body condition score was recorded every two weeks and blood samples were collected periodically during late pregnancy and throughout lactation for analysis of plasma insulin, insulin-like growth factor-1 and non-esterified fatty acid concentrations as previously described [17]. The data for these variables from the specific animals used in the current study are reported to aid interpretation of the animal performance and the transcriptomic results. The data were analysed using SAS version 9.3 (SAS Institute, Cary, NC). All data were tested for normality and log-transformed if necessary. Milk yield, bodyweight and plasma concentrations of insulin, IGF-1 and NEFA were analysed using mixed models procedures with repeated measures. A first-order autoregressive covariance structure was applied, and cow nested within genotype was included as a random effect. Genotype, week, and their interaction were included as fixed effects. The Tukey adjustment was included to correct for multiple comparison tests. The BCS data was analyzed using 
generalized mixed model procedures using a similar model, but because BCS data is ordinal, a multinomial distribution and a cumulative logit link function were specified. None of the animals were bred during the lactation period in which the samples we collected. Hence, there is no confounding effect of pregnancy status on any of the observed phenotypes.

\section{Tissue sampling and RNA extraction}

Tissue biopsies were collected at three time-points relative to parturition (day 0): late pregnancy (LP), day -18 $(\mathrm{sd}=7)$; early lactation (EL), day $1(\mathrm{sd}=1 ; \mathrm{EL})$; and mid-lactation $(\mathrm{ML})$, day 147 (sd $=13)$. Liver tissue was collected by puncture biopsy as previously described [18]. To collect muscle tissue, a biopsy site on the semitendinosus muscle was shaved and sanitized with $7.5 \%$ iodinated povidone and methylated spirits. A subcutaneous injection of lidocaine hydrochloride (2\%) was used to anesthetize the area. An incision was made through the skin, and the biopsy instrument (Biopsy Punch 33-37, Miltex GmbH, Riethein-Weilheim, Germany) was used to remove a core of muscle tissue. The incision site was sutured and treated topically with Duphacycline aerosol (3.6 \% oxytetracycline hydrochloride: Norbrook Laboratories Ltd., Newry, Northern Ireland). Both liver and muscle tissue biopsies were immediately rinsed in saline, blotted dry, snap frozen in liquid nitrogen and stored at $-80^{\circ} \mathrm{C}$ until RNA extraction.

Total RNA was extracted using a standard Trizol-based method. The tissue sample was weighed and $100 \mathrm{mg}$ cut and homogenized in $3 \mathrm{ml}$ TRI Reagent (Sigma-Aldrich, Dublin) until fully homogenized using a hand-held device. The homogenate was removed to sterile Eppendorf tubes (Eppendorf, UK) and incubated at room temperature (RT) for $5 \mathrm{~min}$; samples were centrifuged at $12,000 \times \mathrm{g}$ for 10 $\min$ at $4{ }^{\circ} \mathrm{C}$, and the supernatant was removed to new sterile tubes. Chloroform was added at $0.2 \mathrm{x}$ the volume of homogenate and incubated at RT for $3 \mathrm{~min}$ and samples were centrifuged at $12,000 \times \mathrm{g}$ for $10 \mathrm{~min}$ at $4{ }^{\circ} \mathrm{C}$. Isopropanol was added at $0.6 \mathrm{x}$ the volume of supernatant, vortexed and centrifuged at $12,000 \times \mathrm{g}$ for $10 \mathrm{~min}$ at $4{ }^{\circ} \mathrm{C}$ to pellet the RNA. The supernatant was discarded, the pellet was washed twice in $99 \%$ ethanol (Sigma-Aldrich, Dublin), and centrifuged at 7,500 $\times$ g for $5 \mathrm{~min}$ at $4{ }^{\circ} \mathrm{C}$. The RNA was re-suspended in $50 \mu \mathrm{l}$ nuclease-free water (Sigma-Aldrich, Dublin). A kit based protocol (RNeasy Plus; Qiagen, UK) was used to clean the total RNA, removing the fraction below 200 bp and any genomic DNA. RNA quality was assessed using the Bioanalyser 2100 (Agilent Technologies, UK) with the RNA Nano chip.

\section{Illumina library preparation, sequencing and alignment} Library preparation was conducted using the Truseq v2 kit (Illumina, UK) following the supplied protocol. The
Bioanalyser 2100 (Agilent Technologies, UK) was used to visually determine quality of the libraries with the DNA Nano chip. Concentration was determined using the KAPA Library Quantification (KAPA Biosystems, USA) qPCR method to allow equimolar pooling of each library. Four pools of 24 libraries were made, the maximum possible based on the number of barcoded adapters available. Each pool was sequenced for $100 \mathrm{bp}$ using a paired-end strategy across 3 separate flowcell lanes on an Illumina HiSeq 2000 (Illumina, USA) to minimize technical variation, resulting in 12 lanes of HiSeq data.

The quality of the sequence data was assessed using the FastQC package [23]. Before alignment, sequence 'reads' were trimmed using Trimmomatic with 'trailing' bases (i.e. those at 3 ' end) under a Phred score of 30 being removed [24]. Alignment of reads to the Bos taurus Ensembl70 UMD_3.1 genome [25] was conducted using STAR aligner under default settings with the exception that '-outFilterMismatchNoverLmax' was set to 0.02 . This was because reads were trimmed dynamically to remove by Phred scores, reads were of different lengths and using the ratio of 0.02 mismatches per $x$ bp meant reads were treated equally (version 2.2.0; [26]). Samtools 'view' method was used with the $-\mathrm{f} 2$ flag to return only primary alignments in proper pairs i.e. where the SAM flag was set to 83 and 163, or 99 and 147 for paired reads (version 0.1.18; [27]) and with the -b flag to convert from SAM to BAM files, required to construct count data. Before and after our primary alignment filtering, Samtools'flagstat' was used to determine the numbers of reads aligning to the reference. Counts were determined using the featureCounts utility of the Subread aligner specifying that only reads with pairs on the same chromosome (flags - $\mathrm{p},-\mathrm{P},-\mathrm{C}$ ), and with a maximum insert size of $590 \mathrm{~kb}$ (flag -D) could be used [28]. Counts were defined as the number of times a read-pair mapped to a single annotated feature (gene) of the genome.

\section{Pre-processing of count data}

Count data from featureCounts were filtered before analysis to remove lowly expressed genes using an in-house method [29]. Firstly, all genes with no counts across all samples were removed. Samples were then split by condition and time-point (i.e. 8 samples per group) and calculations of fragments per kilobase per million reads (FPKM; [30]) were used to determine the level of gene expression. FPKM were calculated in R 3.0.1 statistical software [31] using the formula:

$$
\frac{\left(10^{9} \times C\right)}{(N \times L)}
$$

where $C=$ normalized counts per gene, $N=$ total counts per sample, $L=$ gene length. The distribution of 
the mean of the logs $($ base $=\exp (1))$ of the FPKM values (mean $\log (\mathrm{FPKM}))$ per gene in each condition was determined. Any genes found in the lowest $20 \%$ in both conditions were removed from the dataset.

\section{Differentially expressed genes Differential expression analysis}

Filtered counts were used to determine differential expression (DE) of genes using EdgeR Bioconductor package $[32,33]$ under the common dispersion method. The count data was normalized using the trimmed mean of M (TMM) method in the EdgeR [34]. Gene expression differences between the Fert + and Fert- cows were determined at each time-point sampled (LP, EL and ML) resulting in $3 \mathrm{DE}$ gene sets; these are called 'contrasts' in the EdgeR documentation [35]. A false discovery rate (FDR; [36]) of $P<0.05$ was used to correct for multiple testing in all contrasts. Those genes found significantly $\mathrm{DE}$ were retained for further investigation.

\section{Temporal differential expression profiling}

To determine if genes found DE in one or more timepoints had similar expression patterns across timepoints (temporally), the $\operatorname{logFC}$ between genotypes at each time-point was used. If a gene was found DE at any time-point, $\operatorname{logFC}$ was tabulated to give an expression profile for the gene across LP, EL and ML. We indicated the temporal expression profile at each of the three time-points combined using 0 for not DE, and 1 or -1 for DE and up- or down-regulated in Fert + cows respectively. For example, a profile of 0,0,1 indicates not DE in LP, not DE in EL and DE up-regulated in Fert + in ML. A matrix was then constructed showing total genes per profile. From this we used a table to visualize numbers of genes with particular profiles. We refer to these as DE profiles in the following sections.

\section{Functional annotation}

Genes found DE were used as input for the Database for Annotation, Visualization and Integrated Discovery (DAVID) [37, 38], which is a gene set enrichment method using a modified Fisher Exact test. For the 'background' gene set, we used the full set of genes for each contrast following the FPKM filtering step. 'Functional Annotation Clustering' analysis was conducted with default settings including the default $p$-value. The resulting table was downloaded. Each 'Functional Annotation Cluster' contains sets of genes with multiple, mostly similar, annotations (e.g., 'cytoskeleton' or 'microtubule complex') from databases such as the Gene Ontology [39], Interpro [40] and KEGG [41].

\section{Results and Discussion}

\section{Characterization of the animals}

Milk production, bodyweight, BCS and plasma concentrations of insulin, IGF1 and NEFA are illustrated in Fig. 1. Milk energy output and bodyweight were similar, but Fert + cows maintained greater BCS throughout lactation. There was an overall effect of genotype on circulating insulin and IGF1 concentrations, but circulating NEFA concentrations did not differ. These phenotypes for the animals that had biopsies collected for the current study are similar to our previous reports with greater numbers of animals [17-19].

\section{Overview of differential expression}

We found 402, 338 and 282 genes DE in liver and 262, 527 and 212 genes DE in muscle at LP, EL and ML respectively (Additional files 1 and 2). Functional annotation clustering analysis using DAVID identified annotation terms for $26 \%, 25 \%$ and $30 \%$ of the total DE genes in liver and $39 \%, 43 \%$ and $44 \%$ of the $\mathrm{DE}$ genes in muscle from LP, EL and ML, respectively (Additional files 1 and 2). Sequence data metrics are available in Additional file 3: Table S3.

\section{Temporal differential expression profiling}

From the totals of 807 and 815 unique genes found to be $\mathrm{DE}$ in at least one time-point, $79 \%$ and $83 \%$ in liver and muscle, respectively, were up- or down-regulated at one time-point only (see Tables 1 and 2). In liver the top five $\mathrm{DE}$ profiles represented only a single time-point, and contained $12-18 \%$ of all DE genes each. In muscle the two profiles with the most DE genes represented 25 $\%$ of all DE genes each, and were up- or down-regulated only at EL (DE profile $0,1,0$ or $0,-1,0$ ). This observation of time-point specific DE genes indicates that the timepoints are relatively independent from each other in terms of DE gene profiles. Those DE genes found only at a single time-point are likely involved in distinct physiological processes specifically related to that timepoint. This is not too surprising given that the timepoints selected represent distinct phases in the cow's gestation-lactation cycle.

The remaining $21 \%$ and $17 \%$ of the DE genes in liver and muscle, respectively, were therefore found at more than one time-point. This may indicate processes that are systemic or chronic. Interestingly, 23 genes in liver and 17 genes in muscle were found to be up-regulated in Fert + cows at every time-point, and 17 genes in liver and 24 genes in muscle were found to be downregulated in the Fert + animals at every time-point; these may represent dysregulated gene sets in Fert- animals.

Correlation plots of gene expression values in FPKM for each time-point versus the others to indicate concordance 

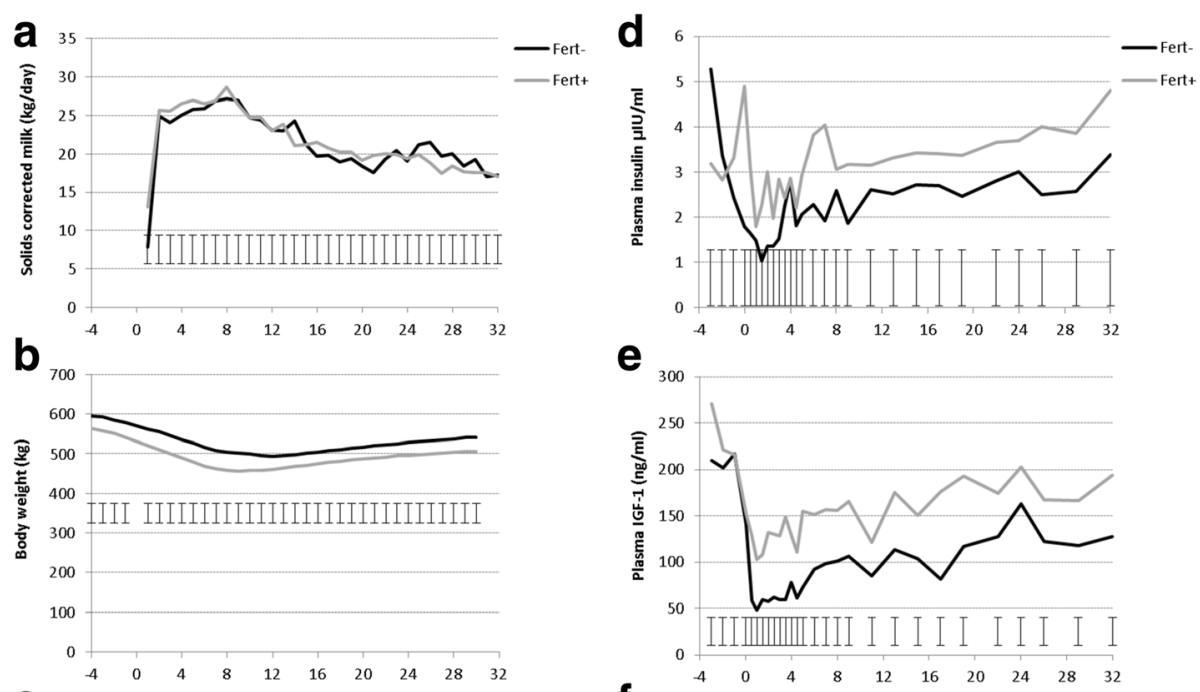

e
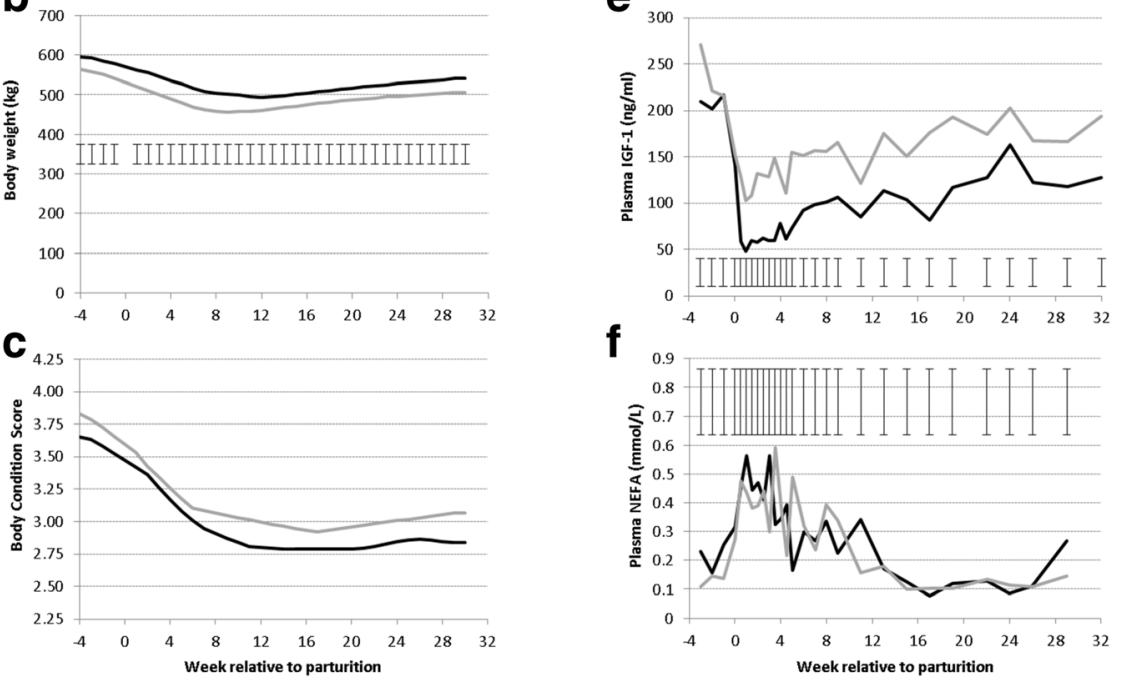

Fig. 1 Phenotypic characterization of the Fert + and Fert- cows. Solids corrected milk yield (a) and bodyweight (b) were similar in both genotypes $(P>0.9$ and $P>0.3$, respectively), but BCS (c) was greater $(P<0.001)$ in Fert + cows compared with Fert- cows. Plasma insulin (d) and IGF1 (e) were greater in Fert + cows compared with Fert- cows $(P=0.003$ and $P<0.001$, respectively), but plasma NEFA concentrations $(\mathbf{f})$ were not different $(P=0.9)$

are reported in Additional file 4: Figures S1(a-f). Pearson correlation $(\mathrm{R})$ value is included in each plot.

\section{Functional annotation}

Two biological themes were apparent from the functional annotation of DE gene sets. The first theme,

Table 1 Liver temporal expression profile

\begin{tabular}{|c|c|c|c|c|c|c|}
\hline & & & EL & & & \\
\hline & & 0 & 1 & -1 & & \\
\hline & & - & $144(0.18)$ & $94(0.12)$ & 0 & \\
\hline & 0 & $41(0.05)$ & $3(0.004)$ & 0 & 1 & \\
\hline & & $106(0.13)$ & $2(0.002)$ & $15(0.02)$ & -1 & \\
\hline & & $122(0.15)$ & $15(0.02)$ & $2(0.002)$ & 0 & \\
\hline \multirow[t]{5}{*}{$L P$} & 1 & $41(0.05)$ & $23(0.03)$ & 0 & 1 & ML \\
\hline & & $11(0.01)$ & $1(0.002)$ & $2(0.004)$ & -1 & \\
\hline & & $129(0.16)$ & $3(0.004)$ & $16(0.02)$ & 0 & \\
\hline & -1 & 0 & 0 & 0 & 1 & \\
\hline & & $19(0.02)$ & $1(0.002)$ & $17(0.02)$ & -1 & \\
\hline
\end{tabular}

All possible profiles across the three time-points and numbers of genes found with those profiles with proportion of total in parentheses; bold indicate profiles where only one time-point contained a DE gene 'immune and inflammatory' processes, was identified from our annotation terms in both liver and muscle. This included time-point specific annotation terms 'chemokine' and 'MHC complex' in LP, 'defense response' and 'immunoglobuiln' in EL, and 'acute phase response' in ML. Of particular note, in this annotation multiple DE

Table 2 Muscle temporal expression profile

\begin{tabular}{|c|c|c|c|c|c|c|}
\hline & & & $\overline{E L}$ & & & \\
\hline & & 0 & 1 & -1 & & \\
\hline & & - & $202(0.25)$ & $207(0.25)$ & 0 & \\
\hline & 0 & $30(0.04)$ & $12(0.01)$ & $1(0.001)$ & 1 & \\
\hline & & $80(0.1)$ & $5(0.01)$ & $16(0.02)$ & -1 & \\
\hline & & $99(0.12)$ & $23(0.03)$ & $3(0.004)$ & 0 & \\
\hline \multirow[t]{5}{*}{$L P$} & 1 & $11(0.01)$ & $17(0.02)$ & 0 & 1 & ML \\
\hline & & $2(0.002)$ & $1(0.001)$ & 0 & -1 & \\
\hline & & $53(0.07)$ & $3(0.004)$ & $13(0.02)$ & 0 & \\
\hline & -1 & $2(0.002)$ & 0 & 0 & 1 & \\
\hline & & $11(0.01)$ & 0 & $24(0.03)$ & -1 & \\
\hline
\end{tabular}

All possible profiles across the three time-points and numbers of genes found with those profiles with proportion of total in parentheses; bold indicate profiles where only one time-point contained a DE gene 
genes were found globally (i.e., across all time-points) and always in the same direction of expression (i.e., upregulated or downregulated in Fert + cows). This indicates that the identified genes (and the resulting annotation) are unlikely to be false-positives.

The second theme was 'metabolism, lipid and carbohydrate' related functions, including 'gluconeogenesis' and 'extra-cellular growth factor' in LP, 'biosynthetic process', 'lipid lipoprotein' and 'metabolic process' in EL, and 'lipid' and 'lipoprotein particle' at ML. There were also several interesting time-point specific functional annotation groups: 'cell cycle/mitosis' in LP, 'tubulin' and 'tRNA' in EL in liver, and 'actin/myosin' and 'tubulin' in EL in muscle.

\section{Global immune and inflammatory related gene annotations}

Global differential expression of genes related to immune and inflammatory processes between Fert + and Fert- cows is a striking result in terms of reproductive efficiency. Genes down-regulated in Fert + cows included bovine leukocyte antigens $B O L A-D Q A 2$ and $B O L A-D Q A 5$ in both tissues as well as BOLA and HLA$D Q B 2$ in muscle. BOLA-DQA1 and MHC class-1 JSP.1 were up-regulated in muscle at every time-point in Fert + cows, and in liver both genes were up-regulated at both LP and ML. The 'DQ' genes are involved in pathogenpresentation to CD4(+) T-lymphocytes [42] and polymorphisms in these genes are involved in disease resistance in cattle [43]. While it was not possible to determine the cause of the immune responses observed, the allocation of resources towards these processes could have a systemic effect on the cows.

The solute carrier of carnitine, SLC22A16, was DE and down-regulated at all time-points in liver. Carnitine has recently been described as a general inflammatory response marker in dairy cows [44] and has been investigated in diet-induced inflammation. It may also be a marker for mitochondrial disease caused by increased circulating fatty acids in obesity [45]. Other mitochondrial-related genes were found globally downregulated in liver, including cytochromes $7 \mathrm{C}$ (COX7C), $C Y B 5 R 2$ and interferon-stimulated gene ISG12(B), which may reside in the mitochondrial membrane and has been shown to mediate caspase-dependant apoptosis in humans [46]. One member of the caspase family, CASP16 (a paralog of CASP6), was also found down-regulated. In muscle, three glutathione S-transferases, GSTM2, GSTM3 and GSTT1, were globally down-regulated. These genes are activated by reactive oxygen species (ROS), and are necessary for clearing toxic oxidized glutathione from the cell [47]. This may be relevant to inflammatory processes, and therefore immune regulators are likely to respond.

\section{Time-point based assessment of annotations Late pregnancy}

'Immune and inflammatory' related An interesting group of genes found up-regulated at LP in liver included an ortholog of the complement factor $\mathrm{H}$ (ENSBTAG00000024647 found DE at approx. one fifth the expression level of CFH, ENSBTAG00000039995), $\mathrm{CFH}$ receptor 4 (CFHR4), which was the most abundant $\mathrm{DE}$ gene, and complement factor D $(C F D)$. These genes represent an immunological response to disease, and also to the products of apoptosis and other cellular debris [48]. Differences in expression may indicate that Fert + cows have greater ability to regulate inflammation compared with Fert- cows at this timepoint.

'Metabolism, lipid and carbohydrate' related Functional annotation groups of the genes DE in liver during LP revealed the annotation term 'gluconeogenesis'. This group included pyruvate carboxylase $(P C)$, serine dehydratase $(S D S)$, fructose-1,6-biphosphatase 2 (FBP2) and histidine decarboxylase $(H D C)$, which were all downregulated in Fert + animals (i.e., more highly expressed in Fert- animals). The role of $P C$ is to convert pyruvate to oxaloacetate using lactate or amino acids (especially alanine, glycine, serine, cysteine and tryptophan) as a precursor, eventually resulting in glucose production. During periods of negative energy balance, the 'labile protein reserve' of skeletal muscle is a significant source of amino acids for hepatic gluconeogenesis [14]. This suggests that Fert- cows may have begun using body reserves for gluconeogenesis earlier than Fert + cows [5]. Further evidence for this comes from both the liver and muscle at LP.

The enzyme pyruvate dehydrogenase kinase 4 (PDK4) phosphorylates pyruvate dehydrogenase, thereby inhibiting conversion of pyruvate to acetyl coA. Greater tissue expression of PDK4 has been implicated in switching to use of NEFAs for cellular energy requirements rather than use of pyruvate [49]. In our study PDK4 was downregulated in both liver and muscle (and was expressed at very high levels in the latter in Fert- animals, see Additional file 1: Table S1). It has been hypothesised that PDK4 might be a 'lipid-status' responsive gene that facilitates pyruvate conservation. Increased PDK4 expression mirrors lipid mobilization from adipose under starvation conditions [50]. This may indicate greater reliance on mobilised NEFA for cellular energy requirements in Fert- cows. In further support of this, fatty acid binding protein $F A B P 4$, which was up-regulated in Fert- cows in muscle, has been shown to have lipolytic activity in skeletal muscle [51]. Thus the increased $P C$ and PDK4 expression in Fert- animals may be the signature of muscle breakdown to provide amino acids for oxaloacetate 
production, as well as use of adipose and intramuscular fat for energy requirements. As this time-point is 3 weeks prepartum, however, it may be mistimed. The expression pattern observed indicates that Fert- cows might have initiated body reserve usage before Fert + cows, which may have a long term impact on their BCS further along in lactation.

At LP in muscle, we found the transforming growth factor-beta-inducible early growth response protein TIEG1 up-regulated. This protein has been implicated in two key cellular processes: (i) apoptosis of cells damaged as a result of mitochondrial beta-oxidation of fatty acids during NEB; and (ii) inhibiting cell proliferation [49]. Other upregulated genes with a functional role in this area include enoyl-coA hydratase $E C H D C 2$, which is involved in the second step of beta-oxidation producing NADPH and acetyl CoA, and the fatty acid desaturase FADS3, the function of which is still not well understood but is known to be regulated by the presence of LCFA [52].

Other genes were annotated as having 'mitochondrial' functions including carnitine palmitoyl transferase $(C P T 1 B)$, cytochrome oxidase $C O X 7 C$ and the calciumbinding mitochondrial solute carrier SLC25A25, all of which were down-regulated, whereas two orthologs of $C O X 7 B$ were both up-regulated. The suppressor of cytokine signalling SOCS2 and insulin-like growth factor binding protein IGFBP1, both down-regulated, attenuate growth hormone $(G H)$ signalling under fasting conditions [53]. Although LP is not a period of fasting, voluntary feed intake has been shown to decline in dairy cattle as parturition approaches, especially during the final week prepartum [8]. A shift towards earlier usage of labile body reserves in Fert- animals during LP was supported by our results, highlighting an aberration in energy metabolism that warrants further study. Further support for this was found with lipin 1 (LPIN1), down-regulated, which is involved in regulation of lipid metabolism by formation of a transcriptional regulation complex with peroxisome proliferator-activated receptor (PPAR) pathway genes PPARA and PGC-1A [54]. Circulating NEFA and glucose concentrations were not different at this time-point, however (see Fig. 1 and [17]).

\section{Early lactation}

\section{'Immune and inflammatory' related}

Alpha-1-acid glycoprotein 1 (AGP1; also called orosomucoid-1 (ORM1)) is a key gene involved in immune and inflammation regulation and response. AGP1 was down-regulated in liver at EL. This gene was the third most abundant transcript at EL using mean FPKM values. Functionally, $A G P$ is an acute-phase plasma protein. In dairy cows, concentrations in blood increase with the severity of uterine bacterial infection and decline during uterine involution [55]. This is consistent with previous observations of the more favourable early postpartum uterine health status in Fert + cows compared with Fert- cows [19]. Additional genes involved in the acute phase response that were down-regulated included serum amyloid A3 (SAA3) and mammary serum amyloid A3.2 (MSAA3.2). SAA3 expression is induced in endometrial epithelial cells in response to challenge with Escherichia coli [56]. Chemokines that were downregulated include CCL3, CCL4, CCL21 and CXCL1O. These chemokines are known to play a functional role in the inflammatory response $[57,58]$. Mannose-binding lectin $(M B L 1)$, also down-regulated, is important in bacterial recognition and innate immunity in cattle. A study using quantitative PCR assays reported elevated hepatic MBL1 mRNA levels in cows with clinical mastitis compared with healthy cows, leading to the conclusion that MBL1 may contribute to resistance to bacterial infection [59].

Activins, inhibins and follistatins are classically known for their role in regulating the synthesis, release and bioactivity of follicle stimulating hormone. These proteins are also involved in inflammation and immune response in many tissues. Inhibin $\mathrm{E}(I N H B E)$, which is the precursor of activin bE, was found to be up-regulated at EL in liver. Activin bE has a high level of similarity to activin A, and can also bind its targets [60]. Activin A is well established as a pro-inflammatory mediator at lower levels of expression, but once inflammation is established and activin A levels rise, an inhibitory effect is seen [61]. Activins are bound by the binding proteins alpha-2-macroglobulin and follistatins. We found the follistatins FST and FSTL1 up-regulated at EL. These genes are implicated in inhibition of activins, and therefore might allow inflammation to proceed [61]. There may be an interesting feedback system operating given that we have found both the potentially anti-inflammatory agent $I N H B E$ and its follistatin regulators up-regulated at EL.

Three genes annotated as tumour necrosis factor receptors were up-regulated (TNFSR6D, TNFSR10B, TNFSR12A). These genes are involved in regulating inflammation, in particular during the induction of apoptosis [62]. Multiple S100A gene family members known to play multiple roles in inflammatory processes were down-regulated in Fert + animals (S100A4, S100A8, S100A9, S100A12 and S100A13). In particular, the calgranulins (S100A8, S100A9, and S100A12) combine to form calprotectin, which is anti-inflammatory and reduces oxidative damage by reactive oxygen species (ROS) in hepatocytes [63]. Also of note, the superoxide dismutase SOD2 was up-regulated. SOD2 is a mitochondrial anti-oxidant and is a nucleoid constituent with mitochondrial DNA (mtDNA) to protect against damage by ROS that might otherwise cause mitochondrial dysfunction [64]. Collectively, these findings suggest that the Fert + cows had greater antioxidant capacity during 
early lactation, which is particularly important at this time to combat increased metabolism-related production of ROS (e.g. oxidation of fatty acids).

\section{'Metabolism, lipid and carbohydrate' related}

We found genes that suggested increased potential for gluconeogenesis at EL in liver. All of the genes discussed herein were up-regulated unless otherwise specified. Phosphoglycomutase (PGM3) converts glucose-1-phosphate to glucose-6-phosphate in glycogenolysis, facilitating conversion of stored glycogen to glucose if required [65]. Asparagine synthase (ASNS) converts aspartate to asparagine, which can then be converted to oxoaloacetate (the rate limiting compound in gluconeogenesis) for use in the citric acid cycle [66]. Further on in that cycle, succinyl-CoA is converted to succinate by succinyl-CoA synthetase (SUCLA2). Serine is a glucogenic amino-acid [67]; the final step of serine production from phosphoserine is controlled by the enzyme phosphoserine phosphatase (PSPH).

In muscle, annotation terms 'lipid lipoprotein', 'metabolic process' and 'amine process' all had multiple upregulated DE genes involved in glucose and fatty acid metabolism. These genes included: serine dehydratase $(S D S)$, which is involved in serine metabolism; phenylethanolamine $\mathrm{N}$-methyltransferase $(P N M T)$, which is involved in norepinephrine conversion to epinephrine; low-density lipoprotein receptor $(L D L R)$; protein kinase gamma PRKAG3; pyruvate kinase PKM2; phosphoglycerate mutase $P G M A 2$; pyruvate dehydrogenase $P D P 2$; the peroxisome proliferator-activated receptor co-activator PPARGC1A; ECHDC2; enolase ENO3; glycerol-3phosphate dehydrogenase 1 (GDP1) and lactate dehydrogenase $(L D H A)$. A recent review highlighted the action of PPARGC1A in inducing a variety of genes under fasting conditions including estrogen related receptor gamma ESRRG, which we found up-regulated [53]. We also found glucose transporters GLUT1 and GLUT4 (or SLC2A1 and $S L C 2 A 4)$ up-regulated. Related genes found downregulated include acyl-CoA synthase $A C S L 4$, apolipoproteins $A P O A 2$ and $A P O D$ and monoamine oxidase MAOA.

\section{Mid-Lactation}

\section{'Immune and inflammatory' related}

Multiple acute phase protein genes such as $S A A 1, S A A 2$ and $S A A 3$, haptoglobin $(H P)$ and glutathione peroxidises $G P X 2$ and GPX3 were all down-regulated in liver. Similarly in muscle, acute phase proteins $H P, S A A 1, A G P$ and fibrinogens $F G A, F G B$ and $F G G$ were all downregulated. These genes have been shown to have greater serum protein expression in cows during disease states [68]. These results once more indicate an inherently different ability to regulate inflammation between cows with divergent genetic merit for fertility.

\section{'Metabolism, lipid and carbohydrate' related}

Genes with annotated functions related to 'lipids' confirmed the quantitative real-time PCR work of Cummins and colleagues [18] undertaken on some of the same animals used in this study. In particular, insulin-like growth factor 1 (IGF-1) was up-regulated in liver. Low circulating insulin during early lactation causes uncoupling of the GH-IGF axis. This results in partitioning of glucose away from insulin-responsive muscle and adipose tissue towards the mammary gland, which is not insulinresponsive [69]. As previously outlined [17, 18], greater hepatic IGF-1 expression during $\mathrm{ML}$ is likely a key mechanism responsible for body reserve repletion in Fert + cows.

Other related genes, all of which were down-regulated in liver, included apolipoproteins $A P O A 1$ and $A P O A 4$, the cytochrome p450s $C Y P 11 A 1$ and $C Y P 1 B 1$, previously reported fatty acid binding proteins $F A B P 3$ and $F A B P 4$, $P S P H$, prostaglandin synthases $A K R 1 C 1, P T G F 2 S L$ and PTGDS and sulfotransferase SULT1E1. Apolipoproteins are constituents of plasma lipoproteins, and fatty-acid binding proteins transport lipids for beta-oxidation in mitochondria, especially LCFA (24) [51]. Up-regulated genes in this group included acetyl-CoA carboxylase $A C A C A$, acyl-CoA synthase $A C S S 2$, ceramide synthase CERS6, fatty acid synthase FASN, synthase and reductase of 3-hydroxy-3-methylglutaryl-CoA HMGCS1 and $H M G C S R$, and squalene epoxidase SQLE.

Finally, in muscle we observed multiple DE genes with annotation terms including 'lipid synthesis' and 'glucose metabolism'. Genes of interest that were down-regulated include apolipoproteins $A P O A 5$ and $A P O H, F A B P 4$, glutamate ammonia ligase (GLUL), and diacylglycerol Oacyltransferase 2 (DGAT2). All of these genes appear to be involved in facilitating greater BCS in Fert + cows, although the specific mechanisms have yet to be elucidated. Overall, the trend seems to indicate that genes involved in fatty acid synthesis pathways are up-regulated in liver tissue of Fert + cows at ML, and that genes involved in lipid synthesis in muscle are increased in Fert- animals at the final timepoint.

\section{Conclusion}

The requirement for greater gluconeogenic capacity in early lactation is a well-studied phenomenon, as is the concomitant change in immune function and inflammation [15]. Here we demonstrate that animals genetically and phenotypically divergent for fertility traits exhibit transcriptomic differences in liver and muscle related to both of these processes at three important time-points during the gestation-lactation cycle. These signals are found across every time-point and in both tissues, indicating that this may be a systemic response in these cows. The findings reported here, and the studies of 
others $[8,15,49,70]$, indicate that immune regulation and inflammatory processes are functional at many stages of the gestation-lactation cycle, and are important for dairy cow fertility and reproductive efficiency. In particular, our results indicate that Fert- cows are less capable of regaining control of inflammation following the onset of lactation. Evidence at the mid-lactation time-point where key metabolism and fertility-related genes such as IGF-1 were differentially regulated supports the idea that both immune/ inflammatory and metabolic processes are fundamental to the phenotypic divergence in our model. It is plausible that these processes are linked. It is also plausible that an increased burden of ROS coupled with a chronic inflammatory state (therefore potentially explaining the continuous expression of BOLA genes into ML) results in Fert- cows expending greater resources trying to effectively maintain/ regain body condition compared with Fert + cows.

\section{Additional files}

Additional file 1: Table S1. Liver Differentially Expressed Gene Data; XLSX workbooks with 6 sheets: (i) "1N_vs_1P.sig", (ii) "2N_vs_2P.sig", (iii) "3N vs 3P.sig" contain differentially expressed genes between FertN and FertP cows at time-points 1,2,3 respectively, (iv) "in_all_TP" are genes found DE in all time-points, (v) fpkms_X are the full FPKMs for each gene in the tissue, (vi) "DAVID_annotation_LP_EL_ML_X" are annotations from DAVID per time-point, specifying each term found significantly enriched for genes, and the DE genes with an arrow to indicate direction of dysregulation, down being lower expression in FertP. (XLSX $15 \mathrm{mb}$ )

Additional file 2: Table S2. Muscle Differentially Expressed Gene Data respectively; XLSX workbooks with 6 sheets: (i) "1N_vs_1P.sig", (ii) "2N_vs_2P.sig", (iii) "3N_vs_3P.sig" contain differentially expressed genes between FertN and FertP cows at time-points 1,2,3 respectively, (iv) "in_all_TP" are genes found DE in all time-points, (v) fpkms $X$ are the full FPKMs for each gene in the tissue, (vi) "DAVID_annotation_LP_EL_ML_X" are annotations from DAVID per time-point, specifying each term found significantly enriched for genes, and the DE genes with an arrow to indicate direction of dysregulation, down being lower expression in FertP. (XLSX $15 \mathrm{mb}$ )

Additional file 3: Table S3. Sequencing Metrics; total reads, aligned reads and uniquely mapped reads per sample in each tissue with mean and standard deviation for each category. (XLSX $11 \mathrm{~kb}$ )

Additional file 4: Figure $\mathbf{1}(\mathbf{a}-\mathbf{f})$. Correlation plots of gene expression values in FPKM for each time-point versus all others per tissue. (ZIP 324 kb)

\section{Abbreviations}

BAM, binary alignment/map; BCS, body condition score; $D E$, differentially expressed; EBV, estimated breeding value; EL, early lactation; LP, late pregnancy; $\mathrm{ML}$, mid-lactation; NEB, negative energy balance; NEFA, non-esterified fatty acids; ROS, reactive oxidation species; SAM, sequence alignment/map

\section{Funding}

BM and SC were funded by the Teagasc Walsh Fellowship Scheme. This project was supported by Teagasc (RMIS 6075) and DAFM grant 13/S/528 led by SB. CJC was funded under the Science Foundation Ireland (SFI) Stokes lecturer scheme (07/SK/B1236A).

\section{Availability of data and materials}

The dataset supporting the conclusions of this article is available in the Gene Expression Omnibus (GEO) repository under accession GSE62159 (http://www.ncbi.nlm.nih.gov/geo/query/acc.cgi?acc=GSE62159).

\section{Authors' contributions}

This study was conceived and designed by SB and SC. Sample collection was carried out by SC and SB. Laboratory analyses were carried out by BM. Bioinformatic analysis was carried out by BM and CJC. Manuscript was written by BM with SB and CJC. All authors read and approved the manuscript.

\section{Competing interests}

The authors declare that they have no competing interests.

\section{Consent for publication}

Not applicable.

\section{Ethics approval and consent to participate}

The experimental procedures involving animals on this study were licensed by the Department of Health, Ireland, in accordance with the Cruelty to Animals Act (Ireland 1876) and the European Community Directive 86/609/EEC.

\section{Author details}

${ }^{1}$ Teagasc, Animal \& Grassland Research and Innovation Centre, Grange, Dunsany, Co. Meath, Ireland. ${ }^{2}$ UCD Conway Institute of Biomolecular and Biomedical Research, University College Dublin, Belfield, Dublin 4, Ireland. ${ }^{3}$ Teagasc, Animal \& Grassland Research and Innovation Centre, Moorepark, Fermoy, Co. Cork, Ireland. ${ }^{4}$ Institute of Biological, Environmental and Rural Sciences, Aberystwyth University, Aberystwyth SY23 3FG, UK.

Received: 18 February 2016 Accepted: 14 July 2016

Published online: 11 August 2016

\section{References}

1. Roche JR, Burke CR, Meier S, Walker CG. Nutrition - reproduction interaction in pasture-based systems: is nutrition a factor in reproductive failure? Anim Prod Sci. 2011:51:1045

2. Roche JR, Bell AW, Overton TR, Loor JJ. Nutritional management of the transition cow in the 21st century: a paradigm shift in thinking. Anim Prod Sci. 2013;53:1000-23.

3. Grummer RR, Wiltbank MC, Fricke PM, Watters RD, Silva-Del-Rio N. Management of dry and transition cows to improve energy balance and reproduction. J Reprod Dev. 2010;56(Suppl):S22-8.

4. Esposito G, Irons PC, Webb EC, Chapwanya A. Interactions between negative energy balance, metabolic diseases, uterine health and immune response in transition dairy cows. Anim Reprod Sci. 2014;144:60-71.

5. Aschenbach JR, Kristensen NB, Donkin SS, Hammon HM, Penner GB. Gluconeogenesis in dairy cows: the secret of making sweet milk from sour dough. IUBMB Life. 2010;62:869-77.

6. Schäff C, Börner S, Hacke S, Kautzsch U, Sauerwein H, Spachmann SK, Schweigel-Röntgen M, Hammon HM, Kuhla B. Increased muscle fatty acid oxidation in dairy cows with intensive body fat mobilization during early lactation. J Dairy Sci. 2013;96:6449-60.

7. De Koster JD, Opsomer G. Insulin Resistance in Dairy Cows. Vet Clin North Am Food Anim Pract. 2013;29:299-322.

8. Sordillo LM, Raphael W. Significance of metabolic stress, lipid mobilization, and inflammation on transition cow disorders. Vet Clin North Am Food Anim Pract. 2013;29:267-78.

9. Loor JJ, Everts RE, Bionaz M, Dann HM, Morin DE, Oliveira R, Rodriguez-Zas SL, Drackley JK, Lewin HA. Nutrition-induced ketosis alters metabolic and signaling gene networks in liver of periparturient dairy cows. Physiol Genomics. 2007;32:105-16.

10. Bionaz M, Loor JJ. Ruminant metabolic systems biology: reconstruction and integration of transcriptome dynamics underlying functional responses of tissues to nutrition and physiological state. Gene Regul Syst Biol. 2012;6:109-25.

11. Seo J, Osorio JS, Schmitt E, Corrêa MN, Bertoni G, Trevisi E, Loor JJ. Hepatic purinergic signaling gene network expression and its relationship with inflammation and oxidative stress biomarkers in blood from peripartal dairy cattle. J Dairy Sci. 2014;97:861-73.

12. Bell AW, Bauman DE. Adaptations of glucose metabolism during pregnancy and lactation. J Mammary Gland Biol Neoplasia. 1997;2:265-78.

13. Larsen M, Kristensen NB. Precursors for liver gluconeogenesis in periparturient dairy cows. Anim Int J Anim Biosci. 2013;7:1640-50.

14. Bell AW. Regulation of organic nutrient metabolism during transition from late pregnancy to early lactation. J Anim Sci. 1995;73:2804-19. 
15. Contreras GA, Sordillo LM. Lipid mobilization and inflammatory responses during the transition period of dairy cows. Comp Immunol Microbiol Infect Dis. 2011;34:281-9.

16. Bauman DE, Currie WB. Partitioning of nutrients during pregnancy and lactation: a review of mechanisms involving homeostasis and homeorhesis. J Dairy Sci. 1980;63:1514-29.

17. Cummins SB, Lonergan P, Evans ACO, Berry DP, Evans RD, Butler ST. Genetic merit for fertility traits in Holstein cows: I. Production characteristics and reproductive efficiency in a pasture-based system. J Dairy Sci. 2012;95:1310-22

18. Cummins SB, Waters SM, Evans ACO, Lonergan P, Butler ST. Genetic merit for fertility traits in Holstein cows: III. Hepatic expression of somatotropic axis genes during pregnancy and lactation. J Dairy Sci. 2012;95:3711-21.

19. Moore SG, Fair T, Lonergan P, Butler ST: Genetic merit for fertility traits in Holstein cows: IV. Transition period, uterine health, and resumption of cyclicity. J Dairy Sci 2014

20. Cummins SB, Lonergan P, Evans ACO, Butler ST. Genetic merit for fertility traits in Holstein cows: II. Ovarian follicular and corpus luteum dynamics, reproductive hormones, and estrus behavior. J Dairy Sci. 2012;95:3698-710.

21. Butler ST. Genetic control of reproduction in dairy cows. Reprod Fertil Dev. 2014;26:1.

22. Moore SG, Scully S, Browne JA, Fair T, Butler ST. Genetic merit for fertility traits in Holstein cows: V. Factors affecting circulating progesterone concentrations. J Dairy Sci. 2014;97:5543-57.

23. Andrews S. FastQC: A Quality Control tool for High Throughput Sequence Data. Babraham Institute, Cambridge, UK. http://www. bioinformatics.babraham.ac.uk/projects/fastqc/(2013). Accessed 8 Apr 2013

24. Bolger AM, Lohse M, Usadel B. Trimmomatic: a flexible trimmer for Illumina sequence data. Bioinforma Oxf Engl. 2014;30:2114-20.

25. ENSEMBL. European Molecular Biology Laboratory, European Bioinformatics Institute (EMBL-EBI), Cambridge, UK. http://www.ensembl.org/info/data/ftp/ index.html (2014). Accessed 28 Apr 2013.

26. Dobin A, Davis CA, Schlesinger F, Drenkow J, Zaleski C, Jha S, Batut $P$, Chaisson M, Gingeras TR. STAR: ultrafast universal RNA-seq aligner. Bioinformatics. 2012;29:15-21.

27. Li H, Handsaker B, Wysoker A, Fennell T, Ruan J, Homer N, Marth G, Abecasis G, Durbin R. 1000 Genome Project Data Processing Subgroup: The Sequence Alignment/Map format and SAMtools. Bioinformatics. 2009;25:2078-9.

28. Liao Y, Smyth GK, Shi W. FeatureCounts: an efficient general purpose program for assigning sequence reads to genomic features. Bioinforma Oxf Engl. 2014;30:923-30.

29. Moran B: https://github.com/brucemoran/coinTools/blob/master/filterFPKM. R (2014). Accessed 3 Oct 2014.

30. Mortazavi A, Williams BA, McCue K, Schaeffer L, Wold B. Mapping and quantifying mammalian transcriptomes by RNA-Seq. Nat Methods. 2008;5:621-8.

31. R Core Team. R: A Language and Environment for Statistical Computing. 3.1 edition (2014). https://www.r-project.org/. Accessed 21 Feb 2014.

32. Robinson MD, McCarthy DJ, Smyth GK. edgeR: a Bioconductor package for differential expression analysis of digital gene expression data. Bioinforma Oxf Engl. 2010;26:139-40.

33. Gentleman RC, Carey VJ, Bates DM, Bolstad B, Dettling M, Dudoit S, Ellis B, Gautier L, Ge Y, Gentry J, Hornik K, Hothorn T, Huber W, lacus S, Irizarry R, Leisch F, Li C, Maechler M, Rossini AJ, Sawitzki G, Smith C, Smyth G, Tierney L, Yang JYH, Zhang J. Bioconductor: open software development for computational biology and bioinformatics. Genome Biol. 2004;5:R80.

34. Robinson MD, Oshlack A. A scaling normalization method for differential expression analysis of RNA-seq data. Genome Biol. 2010;11:R25.

35. Chen Y, McCarthy D, Robinson M, Smyth GK. edgeR: differential expression analysis of digital gene expression data User's Guide. 2014. https://www. bioconductor.org/packages/3.1/bioc/vignettes/edgeR/inst/doc/ edgeRUsersGuide.pdf. Accessed 02 May 2014.

36. Benjamini Y, Hochberg Y. Controlling the False Discovery Rate: A Practical and Powerful Approach to Multiple Testing. J R Stat Soc Ser B Methodol. 1995:57:289-300

37. Huang DW, Sherman BT, Lempicki RA. Systematic and integrative analysis of large gene lists using DAVID bioinformatics resources. Nat Protoc. 2009:4:44-57.
38. Huang DW, Sherman BT, Lempicki RA. Bioinformatics enrichment tools: paths toward the comprehensive functional analysis of large gene lists. Nucleic Acids Res. 2009;37:1-13.

39. Ashburner M, Ball CA, Blake JA, Botstein D, Butler H, Cherry JM, Davis AP, Dolinski K, Dwight SS, Eppig JT, Harris MA, Hill DP, Issel-Tarver L, Kasarskis A, Lewis S, Matese JC, Richardson JE, Ringwald M, Rubin GM, Sherlock G. Gene ontology: tool for the unification of biology. The Gene Ontology Consortium. Nat Genet. 2000;25:25-9.

40. Mitchell A, Chang H-Y, Daugherty L, Fraser M, Hunter S, Lopez R, McAnulla C, McMenamin C, Nuka G, Pesseat S, Sangrador-Vegas A, Scheremetjew M, Rato C, Yong S-Y, Bateman A, Punta M, Attwood TK, Sigrist CJA, Redaschi N, Rivoire C, Xenarios I, Kahn D, Guyot D, Bork P, Letunic I, Gough J, Oates M, Haft D, Huang $H$, Natale DA, et al. The InterPro protein families database: the classification resource after 15 years. Nucleic Acids Res. 2015:43:D213-21.

41. Kanehisa M. KEGG: Kyoto Encyclopedia of Genes and Genomes. Nucleic Acids Res. 2000;28:27-30

42. Norimine J, Brown WC. Intrahaplotype and interhaplotype pairing of bovine leukocyte antigen DQA and DQB molecules generate functional DQ molecules important for priming CD4(+) T-lymphocyte responses. Immunogenetics. 2005;57:750-62.

43. Miyasaka T, Takeshima S-N, Sentsui H, Aida Y. Identification and diversity of bovine major histocompatibility complex class II haplotypes in Japanese Black and Holstein cattle in Japan. J Dairy Sci. 2012;95:420-31.

44. Hailemariam D, Mandal R, Saleem F, Dunn SM, Wishart DS, Ametaj BN. Identification of predictive biomarkers of disease state in transition dairy cows. J Dairy Sci. 2014;97:2680-93.

45. Sampey BP, Freemerman AJ, Zhang J, Kuan P-F, Galanko JA, O'Connell TM, Ilkayeva OR, Muehlbauer MJ, Stevens RD, Newgard CB, Brauer HA, Troester MA, Makowski L. Metabolomic Profiling Reveals MitochondrialDerived Lipid Biomarkers That Drive Obesity-Associated Inflammation. PLoS One. 2012;7, e38812.

46. Makovitzki-Avraham E, Daniel-Carmi V, Alteber Z, Farago M, Tzehoval E, Eisenbach L. The human ISG12a gene is a novel caspase dependent and p53 independent pro-apoptotic gene, that is overexpressed in breast cancer: Apoptosis induction by ISG12a. Cell Biol Int Rep. 2013;20:37-46.

47. Hayes JD, Flanagan JU, Jowsey IR. Glutathione transferases. Annu Rev Pharmacol Toxicol. 2005:45:51-88.

48. Kopp A, Hebecker M, Svobodová E, Józsi M, Factor H. A Complement Regulator in Health and Disease, and a Mediator of Cellular Interactions. Biomolecules. 2012;2:46-75.

49. Wathes DC, Clempson AM, Pollott GE. Associations between lipid metabolism and fertility in the dairy cow. Reprod Fertil Dev. 2013;25:48.

50. Holness MJ, Sugden MC. Regulation of pyruvate dehydrogenase complex activity by reversible phosphorylation. Biochem Soc Trans. 2003;31:1143.

51. Fischer H, Gustafsson T, Sundberg CJ, Norrbom J, Ekman M, Johansson O, Jansson E. Fatty acid binding protein 4 in human skeletal muscle. Biochem Biophys Res Commun. 2006;346:125-30.

52. Reardon HT, Hsieh AT, Jung Park W, Kothapalli KSD, Anthony JC, Nathanielsz PW, Thomas Brenna J. Dietary long-chain polyunsaturated fatty acids upregulate expression of FADS3 transcripts. Prostaglandins Leukot Essent Fatty Acids. 2013;88:15-9.

53. Nakamura MT, Yudell BE, Loor JJ. Regulation of energy metabolism by longchain fatty acids. Prog Lipid Res. 2014;53:124-44.

54. Finck BN, Gropler MC, Chen Z, Leone TC, Croce MA, Harris TE, Lawrence Jr JC, Kelly DP. Lipin 1 is an inducible amplifier of the hepatic PGC-1alpha/ PPARalpha regulatory pathway. Cell Metab. 2006;4:199-210.

55. Sheldon IM, Noakes DE, Rycroft A, Dobson H. Acute phase protein responses to uterine bacterial contamination in cattle after calving. Vet Rec. 2001;148:172-5.

56. Chapwanya A, Meade KG, Doherty ML, Callanan JJ, O'Farrelly C. Endometrial epithelial cells are potent producers of tracheal antimicrobial peptide and serum amyloid $\mathrm{A} 3$ gene expression in response to $\mathrm{E}$. coli stimulation. Vet Immunol Immunopathol. 2013;151:157-62.

57. Guan E, Wang J, Norcross MA. Identification of human macrophage inflammatory proteins 1alpha and 1beta as a native secreted heterodimer. J Biol Chem. 2001;276:12404-9.

58. Heinrichs D, Berres M-L, Nellen A, Fischer P, Scholten D, Trautwein C, Wasmuth HE, Sahin H. The chemokine CCL3 promotes experimental liver fibrosis in mice. PLoS One. 2013:8, e66106. 
59. Liu J, Ju Z, Li Q, Huang J, Li R, Li J, Ma L, Zhong J, Wang C. Mannosebinding lectin 1 haplotypes influence serum MBL-A concentration, complement activity, and milk production traits in Chinese Holstein cattle. Immunogenetics. 2011;63:727-42.

60. Hashimoto O, Tsuchida K, Ushiro Y, Hosoi Y, Hoshi N, Sugino H, Hasegawa Y. cDNA cloning and expression of human activin $\beta E$ subunit. Mol Cell Endocrinol. 2002;194:117-22.

61. Hedger MP, de Kretser DM. The activins and their binding protein, follistatin-Diagnostic and therapeutic targets in inflammatory disease and fibrosis. Cytokine Growth Factor Rev. 2013;24:285-95.

62. Moshage H. Cytokines and the hepatic acute phase response. J Pathol. 1997;181:257-66.

63. Sun Y, Lu Y, Engeland CG, Gordon SC, Sroussi HY. The anti-oxidative, antiinflammatory, and protective effect of S100A8 in endotoxemic mice. Mol Immunol. 2013;53:443-9.

64. Kienhofer J, Haussler DJF, Ruckelshausen F, Muessig E, Weber K, Pimentel D, Ullrich V, Burkle A, Bachschmid MM. Association of mitochondrial antioxidant enzymes with mitochondrial DNA as integral nucleoid constituents. FASEB J. 2009;23:2034-44.

65. Greig KT, Antonchuk J, Metcalf D, Morgan PO, Krebs DL, Zhang J-G, Hacking DF, Bode L, Robb L, Kranz C, de Graaf C, Bahlo M, Nicola NA, Nutt SL, Freeze $\mathrm{HH}$, Alexander WS, Hilton DJ, Kile BT. Agm1/Pgm3-mediated sugar nucleotide synthesis is essential for hematopoiesis and development. Mol Cell Biol. 2007;27:5849-59.

66. Richards NGJ, Kilberg MS. Asparagine synthetase chemotherapy. Annu Rev Biochem. 2006;75:629-54.

67. Hetenyi Jr G, Anderson PJ, Raman M, Ferrarotto C. Gluconeogenesis from glycine and serine in fasted normal and diabetic rats. Biochem J. 1988;253:27-32

68. Chan JP-W, Chang C-C, Hsu W-L, Liu W-B, Chen T-H. Association of increased serum acute-phase protein concentrations with reproductive performance in dairy cows with postpartum metritis. Vet Clin Pathol. 2010;39:72-8

69. Butler ST, Marr AL, Pelton SH, Radcliff RP, Lucy MC, Butler WR. Insulin restores $\mathrm{GH}$ responsiveness during lactation-induced negative energy balance in dairy cattle: effects on expression of IGF-I and GH receptor 1A. J Endocrinol. 2003;176:205-17.

70. Loor JJ. Genomics of metabolic adaptations in the peripartal cow. Anim Int J Anim Biosci. 2010;4:1110-39.

\section{Submit your next manuscript to BioMed Central and we will help you at every step:}

- We accept pre-submission inquiries

- Our selector tool helps you to find the most relevant journal

- We provide round the clock customer support

- Convenient online submission

- Thorough peer review

- Inclusion in PubMed and all major indexing services

- Maximum visibility for your research

Submit your manuscript at www.biomedcentral.com/submit 